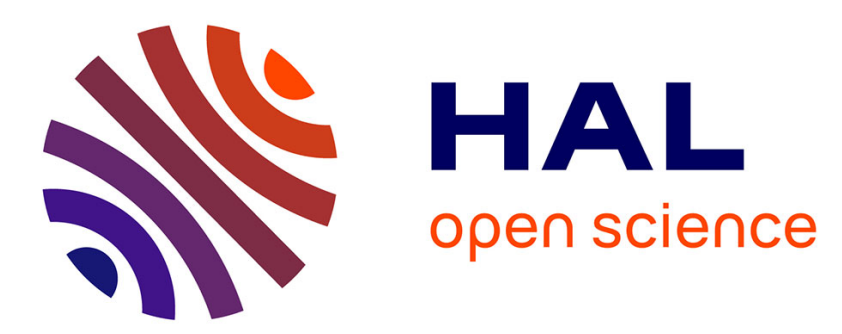

\title{
Heavy-hole intersubband transition of resonantly excited excitons in quantum wells
}

\author{
R. Höpfel, R. Rodrigues
}

\section{To cite this version:}

R. Höpfel, R. Rodrigues. Heavy-hole intersubband transition of resonantly excited excitons in quantum wells. Journal de Physique IV Proceedings, 1993, 03 (C5), pp.C5-15-C5-18. 10.1051/jp4:1993503 . jpa-00251501

\section{HAL Id: jpa-00251501 https://hal.science/jpa-00251501}

Submitted on 1 Jan 1993

HAL is a multi-disciplinary open access archive for the deposit and dissemination of scientific research documents, whether they are published or not. The documents may come from teaching and research institutions in France or abroad, or from public or private research centers.
L'archive ouverte pluridisciplinaire HAL, est destinée au dépôt et à la diffusion de documents scientifiques de niveau recherche, publiés ou non, émanant des établissements d'enseignement et de recherche français ou étrangers, des laboratoires publics ou privés. 


\title{
Heavy-hole intersubband transition of resonantly excited excitons in quantum wells
}

\author{
R.A. HÖPFEL and R. RODRIGUES \\ Institut für Experimentalphysik, Universität Innsbruck, 6020 Innsbruck, Austria
}

\begin{abstract}
The exciton population in a higher subband of GaAs quantum wells, below the free carrier continuum, is studied by time-resolved luminescence spectroscopy. The lifetime of the exciton formed by an electron of the lowest subband and a heavy hole of the second subband is determined as $130 \pm 20 \mathrm{ps}$, in agreement with theoretical estimations of intersubband scattering based on acoustic phonon emission. The exciton lifetime in the second heavy-hole subband is considerably longer than reported values of the recombination time in the lowest exciton state at $k=0$. These findings lead to novel intersubband laser concepts based on excitons.
\end{abstract}

\section{INTRODUCTION}

Relaxation processes of excitons in quantum wells have been studied recently in numerous experiments [1]. The time-resolved studies of radiative recombination [2-7] and also the transition rates of excitons below the free carrier continuum from the light-hole to the heavy-hole exciton subband [8] are of relevance in the context of this work. Other experiments of intersubband relaxation in quantum wells have been performed exclusively with free electrons.

In this work we present the first time-dependent measurement of an exciton in a higher quantized subband state. The $\left(\mathrm{hh}_{2}-\mathrm{e}_{1}\right)_{1 \mathrm{~s}}$ exciton, formed by electrons of the lowest $(\mathrm{n}=1)$ subband and heavy holes of the second $(n=2)$ subband, is studied by time-resolved luminescence. Our experimental results lead to the conclusion that the exciton lifetime in the second heavy-hole subband is limited by acoustic phonon emission and considerably longer than the shortest reported values of radiative lifetimes of the ground state excitons at $k \approx 0$ in ultrahigh quality samples. The most important condition for achieving intersubband inversion can be then fulfilled in principle by optical excitation of higher subband excitons.

\section{EXPERIMENTS}

The structure used in the experiments was grown by molecular beam epitaxy and consists of 40 periods of $265 \AA$ GaAs wells and $260 \AA$ AlAs barriers. The linewidth of the low-temperature luminescence peak is $0.8 \mathrm{meV}$ [9] indicating a high sample quality. The subband structure has been investigated by photoluminescence excitation spectroscopy (PLE), using a $\mathrm{cW}$ Ti:sapphire laser and detection at the $\left(\mathrm{hh}_{1}-\mathrm{e}_{1}\right)_{1 \mathrm{~S}}$ exciton peak. The spectra are shown in Fig. 1. The peak closest to the ground state is the light hole exciton at $k \approx 0$ labelled $\left(\mathrm{lh}_{1}-\mathrm{e}_{1}\right)_{1 \mathrm{~s}}$. The next peak labelled $\left(\mathrm{hh}_{2}-\mathrm{e}_{1}\right)_{1 \mathrm{~s}}$ is the $1 \mathrm{~s}$ exciton formed by an electron of the lowest subband and a heavy hole of the second subband. The radiative transition (absorption and emission) of this exciton is a forbidden transition in perfectly symmetric wells. The observation is possible due to the built-in electric field [10], which increases the band mixing. The electric field necessary for the observed oscillator strength is calculated [9] as $\sim 3 \mathrm{kV} / \mathrm{cm}$, which is 
consistent with midgap Fermi level pinning at the surface. Also the $\left(\mathrm{hh}_{1}-\mathrm{e}_{1}\right)_{2 S}$ and $\left(\mathrm{hh}_{1}-\mathrm{e}_{1}\right)_{3 S}$ excitons [9] can be seen as well as the step-like absorption edge for free electron-hole pair excitation. It is very important for our experiments that the energy of the $\left(\mathrm{hh}_{2}-\mathrm{e}_{1}\right)_{1 \mathrm{~S}}$ exciton is well below the energy of free electron-hole pairs: the energy difference of the $\left(\mathrm{hh}_{2}-\mathrm{e}_{1}\right)_{1 \mathrm{~S}}$ and the $\left(\mathrm{hh}_{1}-\mathrm{e}_{1}\right)_{1 \mathrm{~S}}$ exciton is $4.5 \mathrm{meV}$ compared to the $\left(\mathrm{hh}_{1}-\mathrm{e}_{1}\right)_{1 \mathrm{~S}}$ exciton binding energy of $6.5 \mathrm{meV}[11]$.

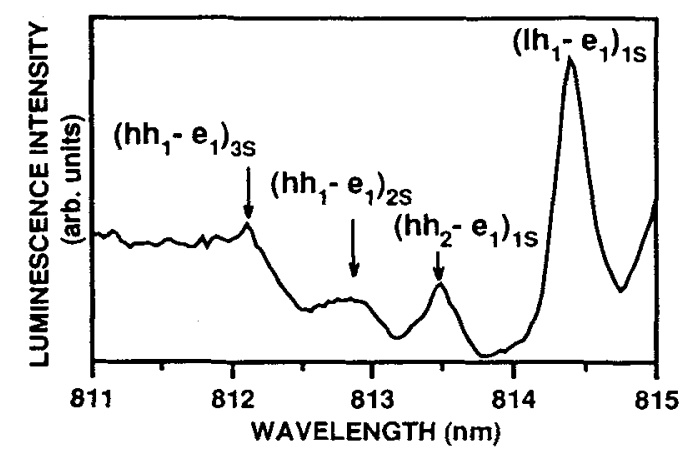

Fig. 1: Excitation spectra obtained with $\mathrm{cw}$ Ti:saphire laser at $\mathrm{T}_{\mathrm{L}}=1.8 \mathrm{~K}$. Detection is at the low-side of the $\left(\mathrm{hh}_{1}-\mathrm{e}_{1}\right)_{1 \mathrm{~s}}$ exciton peak. The various transitions are indicated.

The time-resolved luminescence experiments were performed using a synchroscan streak camera with two-dimensional detection. The excitation was performed by a tunable picosecond dye laser (Styril 8 ) synchronously pumped by a frequency doubled Nd:YAG laser.

For the first series of experiments, with excitation wavelength at $795 \mathrm{~nm}$ and excitation density about $2 \times 10^{9} \mathrm{~cm}^{-2}$ per layer, typical results are shown in Fig. 2a: the amplitude of the $\left(\mathrm{hh}_{2}-\mathrm{e}_{1}\right)_{1 \mathrm{~s}}$ exciton increases to a maximum and decreases at later times. The data points in Fig. $2 b$ are the difference of the total luminescence intensity at the wavelength of the $\left(\mathrm{hh}_{2}-\mathrm{e}_{1}\right)_{1 \mathrm{~S}}$ exciton and the background signal. A first quantitative result is obtained by fitting the experimental data with two time constants for the population and depopulation of the $\left(\mathrm{hh}_{2}-\mathrm{e}_{1}\right)_{1 \mathrm{~S}}$ exciton around $\mathrm{k}=0$. The best fit is shown in the Fig.2b, using values of $140 \pm 30$ and $330 \pm 30$ ps for exponential population and depopulation, respectively. However, as wellknown for rate equations with exponential decays, the two time constants are interchangeable, so the time constant for the depopulation of the $\left(\mathrm{hh}_{2}-\mathrm{e}_{1}\right)_{1 S}$ excitons by intersubband relaxation can be either 140 or 330 ps.

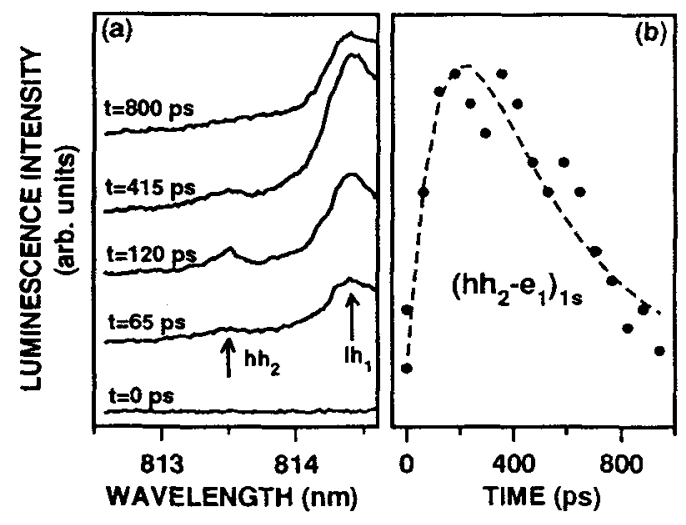

Fig. 2: (a) Time-resolved luminescence data for excitation at $795 \mathrm{~nm}$. $\mathrm{T}_{\mathrm{I}}=1.8 \mathrm{~K}$. (b) $\left(\mathrm{hh}_{2}-\mathrm{e}_{1}\right)_{1 \mathrm{~s}}$ exciton signal as function of time, determined from the difference of the total signal and the background.

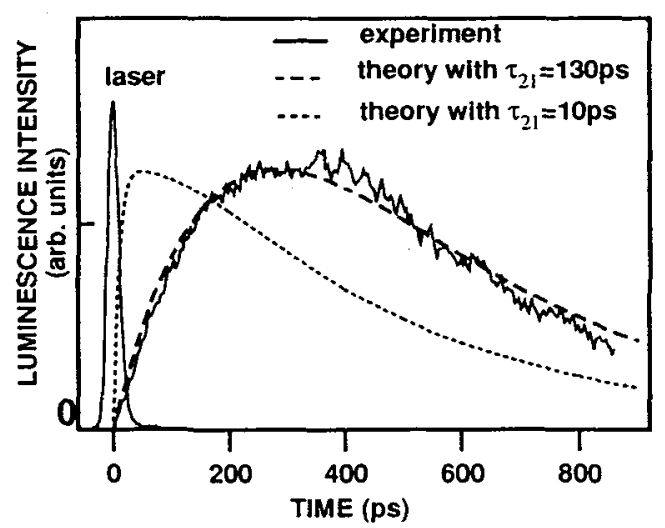

Fig. 3: Time-resolved luminescence signal of the $\left(h_{1}-e_{1}\right)_{1 s}$ exciton after resonant excitation of the $\left(\mathrm{hh}_{2}-\mathrm{e}_{1}\right)_{1 \mathrm{~s}}$ exciton. Dashed lines: theory using $\tau_{21}=130 \mathrm{ps}$ and instantaneous relaxation. 
For a more direct measurement of the $\left(\mathrm{hh}_{2}-\mathrm{e}_{1}\right)_{1 \mathrm{~S}}$ exciton relaxation, we tuned the laser to resonant excitation of the $\left(h h_{2}-e_{1}\right)_{1 S}$ exciton, and studied the time evolution of the luminescence intensity at the $\left(\mathrm{hh}_{1}-\mathrm{e}_{1}\right)_{1 \mathrm{~S}}$ exciton peak. As we can see in the Fig. 3, excitation of the $\left(\mathrm{hh}_{2}-\mathrm{e}_{1}\right)_{1 S}$ exciton causes a rise of the $\left(\mathrm{hh}_{1}-\mathrm{e}_{1}\right)_{1 \mathrm{~S}}$ exciton luminescence much slower than the laser pulse width. The maximum of the luminescence is reached at $\mathrm{t} \approx 350 \mathrm{ps}$, the decay is followed until $\mathrm{t}>800 \mathrm{ps}$.

\section{DISCUSSION}

The quantitative interpretation of this experiment is based on the following model: the laser pulse resonantly excites excitons in the $\left(\mathrm{hh}_{2}-\mathrm{e}_{1}\right)_{1 \mathrm{~s}}$ band around $\mathrm{k} \approx 0$. Intersubband scattering (with a characteristic time constant $\tau_{21}$ ) leads to an increasing population of the $\left(\mathrm{hh}_{1}-\mathrm{e}_{1}\right)_{1 \mathrm{~s}}$ exciton band, where we assume an exponential function for this process. The average excess energy per exciton in the $\left(\mathrm{hh}_{1}\right.$ $\left.\mathrm{e}_{1}\right)_{1 \mathrm{~s}}$ band is about $3.9 \mathrm{meV}$ (corresponding to a temperature of $45 \mathrm{~K}$ ) for acoustic phonon emission as the dominant scattering process. Thermalization is assumed to be much faster than the subsequent energy relaxation, and leads to a thermal distribution in both exciton subbands with one common temperature varying with time. The average energy per exciton is decreased by energy loss via longitudinal acoustic phonon emission in both subbands, according to $[12,8]$ :

$$
\left\langle\frac{d E}{d t}\right\rangle_{L A}=\frac{\left(D_{c}-D_{v}\right)^{2} M_{e x^{2}} 2 k_{B}\left(T_{e x}-T_{L}\right)}{\rho L \hbar^{3}}
$$

where $D_{c}$ and $D_{v}$ are the deformation potentials for electrons and holes [13], $M_{e x}$ and $T_{e x}$ are the exciton mass and temperature, $\rho$ is the mass density and $\mathrm{L}$ is the quantum well width. The average energy is increased by the intersubband scattering and also by the recombination from the $\left(\mathrm{hh}_{1}-\mathrm{e}_{1}\right)_{1 \mathrm{~s}}$ band at $\mathrm{k} \approx$ 0 . Radiative recombination of the $\left(\mathrm{hh}_{1}-\mathrm{e}_{1}\right)_{1 \mathrm{~s}}$ excitons occurs for those excitons with kinetic energy within the homogenous linewidth. Therefore recombination of the $\left(h h_{1}-e_{1}\right)_{1}$ excitons will act as a heating process since only "cold" excitons are removed from the distribution.

From the time-dependence of the luminescence at later times ( $>400 \mathrm{ps}$ ), the radiative recombination time at $\mathrm{k} \approx 0$ can be directly determined from the experiments ( 250 up to $390 \mathrm{ps}$, depending on the excitation intensity). The dependence of the recombination time on the excitation density is in agreement with the recently reported direct measurements using resonant excitation of the ground state excitons [7]. The density dependence is a consequence of the smaller coherence volume at higher densities due to stronger dephasing by exciton-exciton scattering $[2,7]$. With the known recombination time within the homogenous linewidth, the time evolution of the $\left(\mathrm{hh}_{1}-\mathrm{e}_{1}\right)_{1 \mathrm{~S}}$ luminescence can be numerically calculated (Fig. 3, dashed lines).

Using only one variable parameter $\tau_{21}$ for the intersubband relaxation, we obtain the best agreement with our experimental results for a value of $\tau_{21}=130 \pm 20 \mathrm{ps}$ for the intersubband relaxation time from the $\left(\mathrm{hh}_{2}-\mathrm{e}_{1}\right)_{1 S}$ exciton band at $\mathrm{k} \approx 0$ into the $\left(\mathrm{hh}_{1}-\mathrm{e}_{1}\right)_{1 \mathrm{~S}}$ band at $\mathrm{k}>0$. The value of $\tau_{21}$ is identical for both excitation densities and agrees well with the shorter one of the two time constants (140 and 330 ps) resulting from the first series of experiments. In Fig. 3 (dotted line) also the luminescence evolution for very fast intersubband scattering $(10 \mathrm{ps})$ is shown, resulting in a fast rise of the luminescence. This fast rise has been observed experimentally in samples where hot excitons with excess energies within the lowest subband ( $4.8 \mathrm{meV}$ ) comparable to our subband spacing ( $4.5 \mathrm{meV}$ ) have been studied [5]. The delayed luminescence rise observed in our experiments clearly shows the role of intersubband relaxation.

In ref. [14], a theoretical estimate of the intersubband relaxation has been made, calculating the intersubband scattering rate for excitons at $\mathrm{k}=0$ by acoustic deformation potential interaction. For our sample parameters a theoretical value of $\tau_{21}=117 \mathrm{ps}$ is obtained. The slightly longer experimental value might be due to the repopulation of the second subband by the high energy tail of the population in the lowest subband. 
The lifetime of the $\left(\mathrm{hh}_{2}-\mathrm{e}_{1}\right)_{1 S}$ exciton subband is an important parameter for the research focused on intersubband inversion. We find two decisive points that are different for the intersubband dynamics of excitons as compared to free carriers: (1) The intersubband scattering time from the second heavy hole subband $\left(\mathrm{hh}_{2}-\mathrm{e}_{1}\right)_{1 \mathrm{~s}}$ around $\mathrm{k}=0$ is considerably longer (130 ps) than the shortest reported values (40 ps in ref. [5]) of the radiative recombination time in the ground state $\left(h_{1}-e_{1}\right)_{1 S}$ at $k=0$. Theoretical calculations predict even shorter recombination times [4]. Radiative recombination times below 100 ps are expected also for well width above $150 \AA$, if the sample quality is sufficiently high. The intersubband scattering dynamics due to acoustic phonon scattering, however, should not be modified in high quality samples. Thus the most important condition for achieving inversion can be fulfilled in ultrahigh quality samples with large coherence lengths. (2) Since excitons are optically excited only around $k=0$ (in contrast to free electron-hole pairs), the selective excitation of a higher subband is possible without exciting the lower subband at $\mathrm{k}>0$. For these two reasons subband transitions of excitons below the free carrier continuum represent a very appealing new concept for optically pumped coherent sources in the meV range.

\section{ACKNOWLEDGEMENTS}

The experimental work has been performed at Riken (Frontier Research Program), Japan. One of us (R.R.) thanks the Conselho Nacional de Desenvolvimento Científico e Tecnológico (CNPq), Brazil. The work has been supported by the Fonds zur Förderung der wissenschaftlichen Forschung (project \# 8704).

\section{REFERENCES}

[1] For reviews on excitons in quantum wells see, e.g.: S. Schmitt-Rink, D. S. Chemla, and D. A. B. Miller, Advances in Physics 38, 89-188 (1989) and references therein.

[2] Feldmann, G. Peter, E.O. Göbel, P. Dawson, K. Moore, C. Foxon, and R.J. Elliot, Phys. Rev. Lett. 59, 2337 (1987).

[3] E. Hanamura, Phys. Rev. B38, 1228 (1988).

[4] L.C. Adreani, F. Tassone, and F. Bassani, Solid State Commun. 77, 641 (1991).

[5] B. Deveaud, F. Clérot, N. Roy, K. Satzke, B. Sermage, D.S. Katzer, Phys. Rev. Lett. 67,2355 (1991).

[6] Ph. Roussignol, C. Delalande, A. Vinattieri, L. Carraresi, M. Colocci, Phys. Rev. B45, 6965 (1992).

[7] R. Eccleston, B.F. Feuerbacher, J. Kuhl, W.W. Ruihle, and K. Ploog, Phys. Rev. B45, 11403 (1992).

[8] R. Eccleston, R. Strobel, W.W. Rühle, J. Kuhl, B.F. Feuerbacher, and K. Ploog, Phys. Rev. B44, 1395 (1991).

[9] Y. Iimura, Y. Segawa, G.E.W. Bauer, M.M. Lin, Y. Aoyagi, S. Namba, Phys. Rev. B42,1478 (1990).

[10] L. Viña, Surf. Sci. 196, 569 (1988).

[11] R.C. Miller, D.A. Kleinman, W.T. Tsang, and A.C. Gossard, Phys. Rev. B24, 1134 (1981).

[12] T. Takagahara, Phys. Rev. B31, 6552 (1985).

[13] Properties of Gallium Arsenide, 2nd ed., edited by N. Parkman et al., EMIS data reviews

Series No. 2 (IEE and Peter Peregrines LTD., Piscataway, NJ, 1990).

[14] R.A. Höpfel, R. Rodrigues, Y. Iimura, T. Yasui, Y. Segawa, Y. Aoyagi, and S.M. Goodnick, Phys. Rev. B47, 10943 (1993). 